\title{
Pericarditis: A Clinical and Therapeutic Update
}

\author{
Bruno Almeida Santiago ${ }^{1}$, Gabriella Oliveira Moreira帛, Gustavo Silva da Costa ${ }^{1}$, Paulo Ricardo Dantas da \\ Silva ${ }^{1}$, Laura Gomes Lima ${ }^{3}$ and Eriston Vieira Gomes ${ }^{1,2 *}$
}

${ }^{1}$ Morgana Potrich College, FAMP, Medical School, Brazil

${ }^{2}$ Ribeirão Preto Medical School, FMRP, University of São Paulo, Brazil

${ }^{3}$ University of Rio Verde, Medical School, Brazil

*Corresponding author: Eriston Vieira Gomes, Department of Biofunctional, Morgana Potrich College, Mineiros, Brazil.

Received Date: March 28, 2020

Published Date: April 07, 2020

\begin{abstract}
Pericarditis is an inflammatory process that affects the layers of the pericardium. It is characterized by chest pain and electrocardiogram changes and may be followed by pericardial effusion and rub. Here, we aimed to identify the main clinical diagnosis criteria and therapeutic management approaches of pericarditis, its variants, and related complications.
\end{abstract}

Keywords: Pericarditis; Pericardium; Inflammation; Heart disease

Abbreviations: ECG: Electrocardiogram; CRP: C-Reactive Protein; NSAID: Nonsteroidal Anti-Inflammatory Drugs; IRP: Idiopathic Recurrent Pericarditis

\section{Introduction}

Pericarditis is a syndrome caused by pericardial (visceral and parietal pericardia) inflammation and is the most common pericardium-related disease. It may be categorized as acute (symptoms last for less than 4 weeks), recurrent (symptoms persist for 4 and 6 weeks), incessant (symptoms persist for more than 6 weeks), or chronic (symptoms persist for more than 3 months) [13]. Pericarditis is caused due to different factors, such as infectious causes (viral or bacterial, viruses being the most prevalent cause in developed countries and tuberculosis being the most prevalent cause in developing countries), or noninfectious causes, such as myocardial infarction, malignancy, trauma, autoimmune disorders, or idiopathic diseases [2, 4-6].

\section{Clinical Features and Diagnosis}

According to the current European Society of Cardiology guidelines [7], it is necessary to meet at least two of the four defined criteria for diagnosis. The most common criterion is chest pain (which may radiate to the shoulders, arms, and jaw), pericardial effusion (found in $60 \%$ of cases), electrocardiogram (ECG) changes, and pericardial rubs (observed in about 33\% of cases) $[7,8]$.
Moreover, other clinical signals (e.g., elevation in levels of C-reactive protein [CRP], white blood cell count, etc.), and imaging techniques could also be employed for both diagnosis and monitoring of the disease [2,7].

\section{Treatment}

The first-choice therapy for patients with acute and recurrent pericarditis is administration of nonsteroidal anti-inflammatory drugs (NSAIDs) plus colchicine (which shows an anti-inflammatory effect). Considering a non-idiopathic etiology, besides the specific causal treatment (i.e., antimicrobial specific to the etiologic agent), it is recommended that it be associated with an anti-inflammatory agent for symptom control. For specific clinical conditions (see the indicated references), corticosteroids are the next logical choice; however, they have been associated with the risk of relapses $[2,8,9]$. IL-1 blockers (e.g., anakinra) have been used as viable therapeutic agents for autoinflammatory diseases and could potentially be used for treatment of idiopathic recurrent pericarditis (IRP). Immunosuppressive therapy may be recommended for the IRP patients who either are not responsive or exhibit severe side-effects 
to corticosteroids [2, 10-12]. For refractory cases, pericardiectomy should be considered; however, this procedure accompanies $5-10 \%$ mortality rate [1,12]. Exercise restriction is critical and highly recommended for all cases (Figure 1).
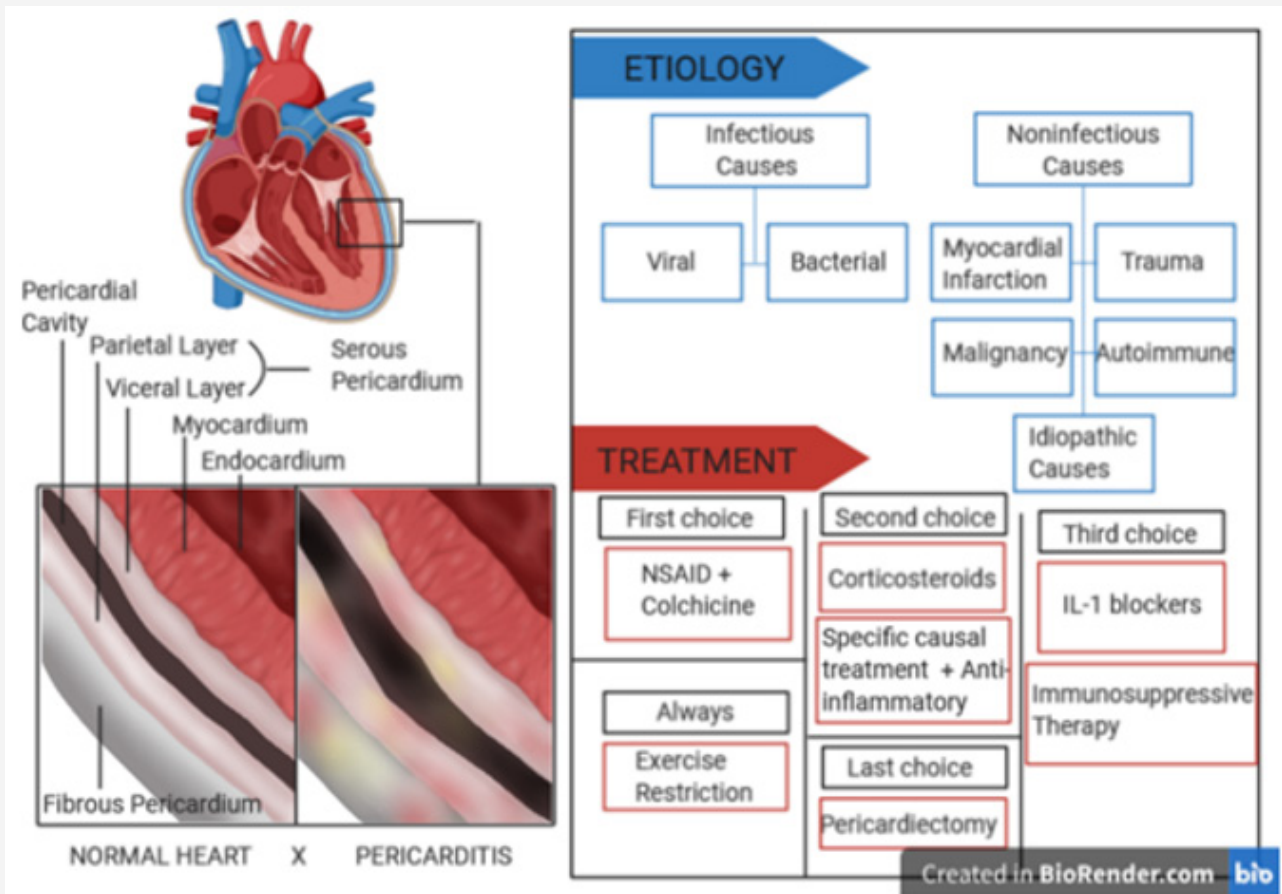

Figure 1: Anatomical, physiological, etiological features, and treatment options for pericarditis. Left side: illustration of a heart and its layers, highlighting a comparison between healthy heart vs. with pericarditis. Upper right: The possible etiologies of pericarditis. Lower right: Possible treatments for different stages of pericarditis.

\section{Complications}

The major pericarditis-related complications include constriction (adhesive or fibrocalcific response, limiting diastolic filling, dissociation of intracardiac and intrathoracic pressures, and heightened ventricular interdependence) and cardiac tamponade (characterized by an equal elevation of atrial and pericardial pressures, pulsus paradoxus [exaggerated expiratory decrease in aortic systolic pressure] and arterial hypotension) [3,13].

\section{Conclusion}

Fast and accurate diagnosis of acute pericarditis, followed by the employment of a safe and efficient treatment approach, may considerably decrease the risk of recurrences and consequent cardiac complications.

\section{Acknowledgment}

None.

\section{Conflicts of Interest}

No conflict of interest.

\section{References}

1. Andreis A, Imazio M, Ferrari GM De (2019) Contemporary diagnosis and treatment of recurrent pericarditis. Expert Rev Cardiovasc Ther 17(11): 817-826.

2. Chiabrando JG, Bonaventura A, Vecchié A, Wohlford GF, Mauro AG, et al. (2020) Management of Acute and Recurrent Pericarditis. JACC 75(1): $76-92$.
3. Cremer PC, Kumar A, Kontzias A, Tan CD, Rodriguez ER, et al. (2016) Complicated Pericarditis Understanding Risk Factors and Pathophysiology to Inform Imaging and Treatment. JACC 68: 2311-2328.

4. Zargar A, Tahir MS, Bhat H (2020) Acute recurrent pericarditis in thyrotoxicosis. IHJ Cardiovasc Case Rep pp. 10-12.

5. Brucato A, Brambilla G, Moreo A, Alberti A, Munforti C, et al. (2006) Long-Term Outcomes in Difficult-to-Treat Patients With Recurrent Pericarditis. Am J Cardiol 98(2): 267-271.

6. Howlett P, Du E, Morrison H, Godsent IC, Wilkinson KA, et al. (2020) The immunopathogenesis of tuberculous pericarditis. Microbes Infect pp. 2-11.

7. Adler Y, Israel C, Brucato A, France PG, Germany KK, et al. (2015) 2015 ESC Guidelines for the diagnosis and management of pericardial diseases - Web Addenda. Eur Heart J 36(42): 2921-2964.

8. Imazio M, Gaita F (2017) Acute and Recurrent Pericardits. Cardiol Clin 35(4): 505-513.

9. Raval J, Nagaraja V, Eslick GD, Denniss AR (2015) The Role of Colchicine in Pericarditis - A Systematic Review and Meta-analysis of Randomised Trials. Hear Lung Circ 24(7): 660-666.

10. Vianello F, Cinetto F, Cavraro M, Battisti A, Castelli M, et al. (2011) Azathioprine in isolated recurrent pericarditis: A single centre experience. Int J Cardiol 147(3): 477-478.

11. Peiffer-Smadja N, Domont F, Saadoun D, Cacoub P (2019) Corticosteroids and immunosuppressive agents for idiopathic recurrent pericarditis. Autoimmun Rev 18(6): 621-626.

12. Dagan A, Langevitz P, Shoenfeld Y, Shovman 0 (2019) Autoimmunity Reviews Anakinra in idiopathic recurrent pericarditis refractory to immunosuppressive therapy; a preliminary experience in seven patients. Autoimmun Rev 18(6): 627-631.

13. Hoit BD (2016) Pathophysiology of the Pericardium. Prog Cardiovasc Dis 59(4): 341-348. 